\title{
Equivariant topological quantum field theory and symmetry protected topological phases
}

\author{
Anton Kapustin ${ }^{a, 1}$ and Alex Turzillo ${ }^{b}$ \\ ${ }^{a}$ Division of Physics, California Institute of Technology, \\ 1200 E California Blvd, Pasadena, CA, 91125 U.S.A. \\ ${ }^{b}$ Simons Center for Geometry and Physics, State University of New York, \\ Stony Brook, NY, 11794 U.S.A. \\ E-mail: kapustin@theory.caltech.edu, aturzillo@theory.caltech.edu
}

ABStRaCt: Short-Range Entangled topological phases of matter are closely related to Topological Quantum Field Theory. We use this connection to classify Symmetry Protected Topological phases in low dimensions, including the case when the symmetry involves timereversal. To accomplish this, we generalize Turaev's description of equivariant TQFT to the unoriented case. We show that invertible unoriented equivariant TQFTs in one or fewer spatial dimensions are classified by twisted group cohomology, in agreement with the proposal of Chen, Gu, Liu and Wen. We also show that invertible oriented equivariant TQFTs in spatial dimension two or fewer are classified by ordinary group cohomology.

Keywords: Topological Field Theories, Discrete Symmetries, Topological States of Matter

ARXIV EPRINT: 1504.01830

\footnotetext{
${ }^{1}$ On leave of absence from California Institute of Technology.
} 


\section{Contents}

1 Introduction and overview 1

2 Oriented equivariant TQFT 3

$2.1 D=0 \quad 3$

$2.2 D=1 \quad 4$

$2.3 \quad D=2 \quad 5$

$3 \quad$ Unoriented equivariant $D=0$ TQFT 6

4 Unoriented equivariant $D=1$ TQFT $\quad 7$

$\begin{array}{lll}4.1 & \text { Definition of unoriented equivariant TQFT } & 7\end{array}$

4.2 Algebraic description for $D=1 \quad 8$

4.3 Invertible unoriented equivariant $D=1$ TQFT 9

$\begin{array}{ll}\text { A Proof of proposition } 1 & 10\end{array}$

B Proof of proposition 2 13

\section{Introduction and overview}

Recently the problem of classifying gapped phases of matter whose ground state is shortrange entangled ${ }^{1}$ (SRE phases) has received a lot of attention. Two gapped local Hamiltonians (or gapped systems) are said to lie in the same gapped phase if there is a continuous family of gapped systems that interpolates between them. In the context of systems with a global symmetry $G$, a phase with symmetry $G$ is defined by requiring the family of Hamiltonians to be symmetric. Gapped phases can be divided into two broad classes, bosonic and fermionic, depending on whether the fundamental degrees of freedom are bosons or fermions. The bosonic SRE phases are in many ways simpler, and there has been a substantial progress in their classification. In particular, it has been proposed in [3] that $D$-dimensional bosonic SRE phases with a finite internal symmetry $G$ are classified by the abelian group $H^{D+1}(B G, \mathrm{U}(1))$. Here $B G$ is the classifying space of $G$, and $D$ is the dimension of space (thus the dimension of spacetime is $D+1$ ). Later it was noticed that some SRE phases in spatial dimension 3 are not captured by the group cohomology classification [4], and it was proposed by one of the authors that the classification can be improved by replacing ordinary cohomology of $B G$ with a particular generalized cohomology theory (the stable cobordism) [5] (see also [1, 6]). For $D \leq 2$ all classification schemes agree. In

\footnotetext{
${ }^{1}$ There are at least two common definitions of short-range entanglement. Here, we follow [1] in defining SRE as invertibility. The notion of an SPT (defined and discussed below) captures the other definition [2].
} 
fact one can use the matrix product representation of SRE states to prove that $D=1$ bosonic SRE phases are classified by $H^{2}(B G, \mathrm{U}(1))$ [7]. The $D=1$ fermionic SRE phases have also been classified [8]. The $D=0$ case is even simpler.

One promising avenue for extending these results to higher dimensions is via equivariant Topological Quantum Field Theory (TQFT). It is an attractive conjecture that a large class of gapped phases is described at large scales by a TQFT. It is widely believed that if the large-volume limit of a quantum system exists, then its long distance behavior is described by an effective field theory. For a gapped system, which has no long wavelength propagating degrees of freedom, the effective theory is a TQFT. Gapped systems in the same phase are expected to share a single long distance effective description, or at least their effective descriptions can be continuously connected. If this is the case, then there is a one-to-one correspondence between gapped phases and deformation classes of TQFTs. ${ }^{2}$

Both gapped phases and TQFTs can be tensored, and each set has a neutral element 1 corresponding to the trivial phase or TQFT. This operation makes each set into a commutative monoid (a set with an associative and commutative binary operation and a unit). An element $\Phi$ of a monoid is said to be invertible if there exists an element $\bar{\Phi}$ such that $\Phi \circ \bar{\Phi}=\bar{\Phi} \circ \Phi=1$. Thus it makes sense to talk about invertible gapped phases and invertible TQFTs. The set of invertible elements forms an abelian group. According to one definition of SRE phases [1], an invertible gapped phase is the same as an SRE phase; that is, a gapped system $\phi$ in a phase $\Phi$ is an SRE if there exists another gapped system $\bar{\phi}$ in $\bar{\Phi}$ such that $\phi \otimes \bar{\phi}$ can be deformed to the trivial (product state) system without closing the gap. If one believes into the correspondence between gapped phases and TQFTs, the classification of SRE phases is reduced to the classification of invertible TQFTs up to a continuous deformation.

Consider now phases with a symmetry $G$. These also form a commutative monoid, and forgetting the symmetry gives us a map to the monoid of all phases. Phases with a symmetry $G$ are mapped to the neutral element under this map are usually called SPT phases. Note that it is not clear from this definition whether SPT phases with a symmetry $G$ are invertible as $G$-symmetric phases, but it is believed to be true. SRE phases with a symmetry $G$ are conjectured to correspond to invertible $G$-equivariant TQFTs.

While classifying TQFTs in $D>1$ is unrealistic, classifying invertible ones is much simpler. In fact, using the known algebraic description of equivariant TQFTs in $D=0$, 1 and 2, it is easy to check that in these dimensions invertible $G$-equivariant TQFTs are classified by $H^{D+1}(B G, \mathrm{U}(1))$, provided the group $G$ does not act on spacetime. But if some elements of $G$ involve time-reversal, the problem is more complicated. From the TQFT viewpoint, time-reversal symmetry means that the theory can be defined on unorientable spacetimes. The difficulty is that an algebraic description of unoriented equivariant TQFTs is not known even in low dimensions. The main goal of this paper is to provide such an algebraic description in $D=0$ and 1 and to show that invertible equivariant TQFTs are classified by twisted group cohomology $H^{D+1}\left(B G, \mathrm{U}(1)_{\rho}\right)$, where $\rho: G \rightarrow \mathbb{Z}_{2}$

\footnotetext{
${ }^{2}$ There are exceptions to this rule, however, due to the existence of phases with non-vanishing thermal Hall conductivity. These only occur in $D=2 \bmod 4$, where there exist gravitational Chern-Simons terms.
} 
is a homomorphism which tells us which elements of $G$ are time-reversing and which are not. This agrees with the proposal of [3]. It is likely that this method can be extended to $D=2$. In higher dimensions an algebraic description of general TQFTs is prohibitively complicated, and this approach to classifying SRE phases becomes impractical. Note that equivariant TQFTs which are not necessarily invertible are interesting in their own right, as they describe Symmetry Enhanced Topological (SET) phases.

In section 2 we deal with the case of a finite symmetry $G$ which acts trivially on spacetime. We recall algebraic descriptions of oriented equivariant TQFTs in $D \leq 2$ and show that invertible equivariant TQFTs are classified by elements of $H^{D+1}(B G, \mathrm{U}(1))$. All of this is either trivial $(D=0)$ or well-known to experts $(D=1$ and 2$)$.

In section 3 we consider unoriented equivariant TQFT in $D=0$ and the corresponding SRE phases with time-reversing symmetries.

In section 4 we formulate axioms of unoriented equivariant TQFT in $D=1$ by extending Turaev's axioms in the oriented case [11]. We show how these axioms lead to a generalization of Turaev's $G$-crossed algebra, which we call $\rho$-twisted $G$-crossed algebra. We prove that every $\rho$-twisted $G$-crossed algebra gives rise to an unoriented equivariant TQFT. Finally we show that invertible TQFTs in $D=1$ give rise to $\rho$-twisted 2-cocycles on $B G$, and that conversely to every element of $H^{2}\left(B G, \mathrm{U}(1)_{\rho}\right)$ one can associate a $\rho$-twisted $G$-crossed algebra which is unique up to isotopy.

It would be interesting to give an algebraic description of $D=2$ unoriented equivariant TQFTs and show that in the invertible case they are classified by $H^{3}\left(B G, \mathrm{U}(1)_{\rho}\right)$. The first step is to categorify our algebraic description of $D=1$ unoriented equivariant TQFT by replacing vector spaces with categories, linear maps with functors, and equalities with isomorphisms. The nontrivial part is to find a complete set of coherence conditions between isomorphisms analogous to the pentagon and hexagon conditions in the oriented case which ensure consistency under gluing.

Since this paper was submitted to the arXiv, there have been several developments. Freed and Hopkins [19] proved a theorem relating invertible unitary TQFTs and stable cobordisms. For $D=1$ it reduces to the statement that invertible unitary equivariant TQFTs are classified by elements of $H^{2}\left(B G, \mathrm{U}(1)_{\rho}\right)$. More recently Bhardwaj [20] generalized the Turaev-Viro construction of equivariant $D=2$ TQFTs to the unoriented case.

A.K. would like to thank V. Ostrik for helpful discussions. The work of A.K. was supported by the Simons Foundation. The work of A.T. was supported in part by the U.S. Department of Energy, Office of Science, Office of High Energy Physics, under Award Number DE-SC0011632.

\section{Oriented equivariant TQFT}

\section{$2.1 \quad D=0$}

A $D=0$ TQFT is ordinary quantum mechanics with zero Hamiltonian and is completely determined by its space of states (a finite-dimensional complex vector space $V$ ). Equivariant TQFT is merely a vector space $V$ with an action of $G$. Since $G$ is finite, this representation is unitarizable (unitary for a suitable choice of inner product on $V$ ). The 
trivial equivariant TQFT corresponds to $V=\mathbb{C}$ with a trivial action of $G$. Equivariant TQFTs which are invertible with respect to the tensor product are one-dimensional representations of $G$, i.e., elements of $H^{1}\left(B G, \mathbb{C}^{*}\right) \simeq H^{1}(B G, \mathrm{U}(1))$.

\section{$2.2 D=1$}

$D=1$ TQFTs are in one-one correspondence with commutative Frobenius algebras [9] (see [10] for a nice exposition, including various generalizations). The vector space $\mathcal{A}$ underlying the algebra is the space of states of the TQFT on a circle. The state-operator correspondence identifies $\mathcal{A}$ with the space of local operators, which is clearly a commutative algebra. The Frobenius structure is a non-degenerate bilinear inner product

$$
\eta(a, b) \in \mathbb{C}, \quad a, b \in \mathcal{A},
$$

satisfying $\eta(a b, c)=\eta(a, b c)$. It is a combination of the usual sesquilinear Hilbert space inner product and the anti-linear CPT transformation:

$$
\eta(a, b)=(\mathrm{CPT} a, b) .
$$

The trivial $D=1$ TQFT has $\mathcal{A} \simeq \mathbb{C}$ and $\eta(1,1)=1$. An invertible TQFT has $\mathcal{A} \simeq \mathbb{C}$, and thus is completely determined by $\eta(1,1) \in \mathbb{C}^{*}=\mathbb{C} \backslash\{0\}$. If we are interested only in classifying TQFTs up to isotopy (i.e. up to continuous deformations), then all these TQFTs can be identified (since $\pi_{0}\left(\mathbb{C}^{*}\right)$ is trivial). If we identify invertible TQFTs and SRE phases, this means that in the absence of symmetry there are no nontrivial $D=1$ SRE phases.

To incorporate a symmetry $G$, we need to consider $G$-equivariant $D=1$ TQFTs. $G$ equivariance means that we can couple the theory to an arbitrary $G$-bundle. The precise definition of equivariant TQFT will be recalled in section 3. For now, we only need the algebraic description of such TQFTs due to Turaev [11]. He defines a $G$-crossed algebra as a Frobenius algebra $\left(\mathcal{A}=\oplus_{g \in G} \mathcal{A}_{g}, \eta\right)$ together with a homomorphism $\alpha: G \rightarrow$ Aut $\mathcal{A}$ such that

$$
\begin{aligned}
& \mathcal{A}_{g} \cdot \mathcal{A}_{h} \subset \mathcal{A}_{g h} \text { and } 1 \in \mathcal{A}_{1} . \\
& \eta\left(\mathcal{A}_{g}, \mathcal{A}_{h}\right)=0 \text { if } g h \neq 1 . \\
& \alpha_{h}\left(\mathcal{A}_{g}\right) \subset \mathcal{A}_{h g h^{-1}} . \\
& \alpha \text { preserves } \eta \text { and }\left.\alpha_{h}\right|_{\mathcal{A}_{h}}=\text { id. } \\
& \forall \psi_{g} \in \mathcal{A}_{g}, \psi_{h} \in \mathcal{A}_{h} \text { we have } \psi_{g} \cdot \psi_{h}=\alpha_{g}\left(\psi_{h}\right) \cdot \psi_{g} . \\
& \forall g \in G \text { let } \xi_{i}^{g} \text { and } \xi_{g}^{i} \text { be dual bases in } \mathcal{A}_{g} \text { and } \mathcal{A}_{g^{-1}} \text {. Then } \\
& \quad \sum_{i} \alpha_{h}\left(\xi_{i}^{g}\right) \xi_{g}^{i}=\sum_{j} \xi_{j}^{h} \alpha_{g}\left(\xi_{h}^{j}\right), \forall g, h \in G .
\end{aligned}
$$

Let us make a few remarks about this definition. $\mathcal{A}_{g}$ is the $g$-twisted sector of the space of states on a circle, and $\alpha_{h}$ describes the action of $G$ on the space of states. If $G$ is abelian, it acts on each twisted sector separately, but in general it mixes different twisted sectors. The penultimate axiom shows that $\mathcal{A}$ is not commutative, but is twisted-commutative. The last axiom arises from considering a punctured torus with twists by $g$ and $h$ along the two 
generators of its fundamental group and computing the corresponding state in two different ways. This axiom, together with the Frobenius condition $\eta(a, b c)=\eta(a b, c)$, implies

$$
\operatorname{dim} \mathcal{A}_{g}=\left.\operatorname{Tr} \alpha_{g}\right|_{\mathcal{A}_{1}}
$$

Both sides of this equality compute the partition function of a torus twisted by $g$ along one direction and by 1 along the other direction. On the left-hand side, the direction twisted by $g$ is regarded as space and the direction twisted by 1 is regarded as time. On the right-hand side, it is the other way around. Since the right-hand side is a character of a finite group $G$, we get an inequality $0<\operatorname{dim} \mathcal{A}_{g} \leq \operatorname{dim} \mathcal{A}_{1}$. That is, twisting by $g$ cannot increase the number of states.

In particular, let us consider an invertible $G$-equivariant TQFT. Then $\mathcal{A}_{1} \simeq \mathbb{C}$, and therefore $\mathcal{A}_{g} \simeq \mathbb{C}$ for all $g \in G$. If we choose a basis vector $\ell_{h}$ in each $\mathcal{A}_{h}$, we see that the algebra structure is given by a collection of complex numbers $b(g, h)$ such that

$$
\ell_{g} \cdot \ell_{h}=b(g, h) \cdot \ell_{g h}
$$

Twisted commutativity of $\mathcal{A}$ implies that $b(g, h)$ is nonzero for all $g, h$ and fixes $\alpha_{h}$ in terms of $b$. Associativity of multiplication implies that $b$ is a 2-cocycle, and changing a basis in $\mathcal{A}_{g}$ changes it by a coboundary. The rest of the axioms are easily checked. With $b$ fixed, the only freedom left is the choice of the inner product $\eta$; all such choices lead to isotopic TQFTs, which means that isotopy classes of invertible oriented equivariant $D=1$ TQFTs are classified by $[b] \in H^{2}\left(B G, \mathbb{C}^{*}\right) \simeq H^{2}(B G, \mathrm{U}(1))$. This result has been proved in [11].

\section{$2.3 D=2$}

When studying oriented $D=2$ TQFTs, one usually assumes that the space of local operators (i.e. the vector space attached to $S^{2}$ ) is one-dimensional, and thus the algebra of local operators is isomorphic to $\mathbb{C}$. If one is interested only in unitarizable TQFTs, one does not lose much by focusing on this special case. Indeed, it is easy to show that if the TQFT is unitarizable (i.e. the bilinear inner product arises from a Hermitian inner product and an antilinear CPT symmetry), the algebra of local operators is semisimple. It is also commutative, and therefore isomorphic to a sum of several copies of $\mathbb{C}$. The generators of this algebra label different superselection sectors, and one might as well focus on a single sector where all but one generator act trivially. The argument applies equally well for all $D>0$, but in $D=1$ it is traditional to allow the algebra of local operators to be non-semisimple, in view of string theory applications which require one to consider non-unitary TQFTs.

We are interested in unitarizable oriented $D=2$ TQFTs, and therefore in this section we assume that the space of local operators is $\mathbb{C}$. Such theories are described by modular tensor categories with vanishing central charge $c \in \mathbb{Z} / 8[12,13]$. (If the central charge is nonzero, one gets a framed $D=2 \mathrm{TQFT}$ ). The data of a modular tensor category attaches a vector space to every closed oriented 2-manifold, and a map of vector spaces to every oriented bordism between such 2-manifolds. Similarly, oriented equivariant $D=2$ TQFT is described by a $G$-modular category $[14,15]$. Its definition is a categorification of the notion of $G$-crossed algebra. In particular, for every $g \in G$ one has a category 
$\mathcal{C}_{g}$, and a bi-functor $\mathcal{C}_{g} \times \mathcal{C}_{h} \rightarrow \mathcal{C}_{g h}$ satisfying the associativity constraint. The data of a $G$-modular category attaches a vector space to every closed oriented 2-manifold with a $G$ bundle and a trivialization at a base point, and a map of vector spaces for every oriented $G$-bordism between such 2-manifolds (i.e. to every oriented 3-manifold with a $G$-bundle which "interpolates" between the two oriented 2-manifolds with $G$-bundles). Objects of the category $\mathcal{C}_{g}$ represent quasi-particles in the $g$-twisted sector.

An invertible oriented equivariant $D=2$ TQFT is described by a $G$-modular category with $\mathcal{C}_{1} \simeq$ Vect, where Vect is the category of finite-dimensional vector spaces. This condition ensures that for the trivial $G$-bundle the vector space attached to any oriented 2-manifold is one-dimensional. If the TQFT describes a gapped phase, its space of ground states is non-degenerate for any topology. This is a hallmark of an SRE phase.

From $\mathcal{C}_{1} \simeq$ Vect one can deduce that $\mathcal{C}_{g} \simeq$ Vect for all $g \in G$. Indeed, by the definition of a $G$-modular category [15], $\mathcal{C}_{g}$ is nonempty for all $g \in G$. Then proposition 4.58 in [16] implies that $\mathcal{C}_{g} \simeq$ Vect. As a consequence, the vector space attached to any 2-manifold with any $G$-bundle is one-dimensional. That is, there is no ground-state degeneracy even after twisting by an arbitrary $G$-bundle.

Finally, proposition 4.61 in [16] tells us that in the invertible case $\mathcal{C}$ is entirely determined by an element of $H^{3}\left(B G, \mathbb{C}^{*}\right) \simeq H^{3}(B G, \mathrm{U}(1))$. This agrees with the proposal of [3] that $D=2$ bosonic SRE phases with symmetry $G$ are classified by elements of $H^{3}(B G, \mathrm{U}(1))$.

\section{Unoriented equivariant $D=0$ TQFT}

A homomorphism $\rho: G \rightarrow \mathbb{Z}_{2}$ encodes whether a particular symmetry $g$ preserves or reverses the direction of time. We identify $\mathbb{Z}_{2}$ with $\{1,-1\}$ and let $\rho(g)=-1$ if $g$ is time-reversing and $\rho(g)=1$ otherwise. Recall that a $\rho$-twisted 1-cochain on $B G$ is the same as a function $\phi: G \rightarrow \mathrm{U}(1)$ satisfying

$$
\phi(g h)=\phi(g) \phi(h)^{\rho(g)} .
$$

Two twisted cochains $\phi(g)$ and $\psi(g)$ are regarded as equivalent (i.e. cohomologous) if there exists $\mu \in \mathrm{U}(1)$ such that for all $g \in G$ we have

$$
\psi(g)=\mu^{\rho(g)-1} \phi(g)= \begin{cases}\phi(g), & \rho(g)=1 \\ \mu^{-2} \phi(g), & \rho(g)=-1\end{cases}
$$

To each $g \in G$ an equivariant $D=0$ TQFT associates an operator on the vector space $V$ assigned to the point:

$$
\Lambda(g): V \rightarrow V
$$

where $\Lambda(g)$ is linear if $\rho(g)=1$ and anti-linear if $\rho(g)=-1$. After choosing a basis in $V$, we can attach to every $\Lambda(g)$ a complex non-degenerate matrix $M(g)$, by letting

$$
\Lambda(g)= \begin{cases}M(g), & \rho(g)=1 \\ M(g) K, & \rho(g)=-1\end{cases}
$$


Here $K: V \rightarrow V$ is an operator which complex-conjugates the coordinates of a vector in the chosen basis. The matrices $M(g)$ do not form a complex representations of $G$, rather [17]:

$$
M(g h)= \begin{cases}M(g) M(h), & \rho(g)=1 \\ M(g) M(h)^{*}, & \rho(g)=-1 .\end{cases}
$$

In the invertible case $V \simeq \mathbb{C}$ the matrices $M(g)$ become elements of $\mathbb{C}^{*}$, and (3.5) becomes precisely the twisted cocycle condition for the $\mathbb{C}^{*}$-valued 1-cochain $M(g)$, where $\mathbb{Z}_{2}$ acts on $\mathbb{C}^{*}$ by complex conjugation.

We should also investigate the effect of a change of basis in $V$. In the invertible case, if we replace the basis element $\ell \in V$ by $\lambda^{-1} \ell, \lambda \in \mathbb{C}^{*}$, the function $M(g)$ transforms as follows:

$$
M(g) \mapsto \begin{cases}M(g), & \rho(g)=0 \\ \lambda^{-1} \lambda^{*} M(g), & \rho(g)=1 .\end{cases}
$$

This is precisely the shift of the twisted 1-cocycle $M(g)$ by a twisted coboundary. Thus equivalence classes of invertible unoriented equivariant $D=0$ TQFTs are classified by elements of the twisted cohomology group $H^{1}\left(B G, \mathbb{C}_{\rho}^{*}\right) \simeq H^{1}\left(B G, \mathrm{U}(1)_{\rho}\right)$.

\section{Unoriented equivariant $D=1$ TQFT}

\subsection{Definition of unoriented equivariant TQFT}

For $D>0$ we can avoid anti-linear operators by interpreting the orientation-reversing symmetry as a parity symmetry ( $P$ or $C P$ ). Since $C P T$ is a symmetry of any local unitary QFT, we do not loose generality by doing this. Thus $\rho(g)=-1$ if $g$ reverses spatial orientation and $\rho(g)=1$ otherwise.

At first we will try to be as general as possible and do not fix the spatial dimension $D$. Consider a finite group $G$ together with a homomorphism $\rho: G \rightarrow \mathbb{Z}_{2}$, and let $G_{0}$ denote the kernel of $\rho$. For any manifold $X$ we will denote by $o(X)$ its orientation bundle. Any TQFT is defined as a functor from a geometric source category with a symmetric monoidal structure to the category of finite-dimensional vector spaces Vect (or more generally, to a symmetric monoidal category).

In the case of equivariant TQFT based on the pair $(G, \rho)$, the source category $\mathcal{C}$ is defined as follows. An object of $\mathcal{C}$ is a closed $D$-manifold $M$, a base point for every connected component of $M$, a $G$-bundle $E$ over $M$, a trivialization of $G$ at every base point, and a trivialization of $o(M) \otimes \rho(E)$ everywhere on $M$. Here, $\rho(E)$ denotes the $\mathbb{Z}_{2}$-bundle given by the quotient of $E \times \mathbb{Z}_{2}$ by $(e, x) \sim\left(e g^{-1}, \rho(g) x\right)$, and the last datum expresses the fact that $\rho(E)$ is isomorphic to the orientation bundle of $X$. A morphism of $\mathcal{C}$ is an isomorphism class of a $D+1$-dimensional bordism $N$ equipped with a $G$-bundle $E$ and a trivialization of $o(N) \otimes \rho(E)$, with every connected component of the boundary given a base point and a trivialization of $E$ there. Two such bundles are said to be isomorphic if they are related by a bundle map that is an homeomorphism of the total space, covers a homeomorphism of the base space, and preserves the trivialization and boundary data. There is also a decomposition of the boundary into two disjoint parts, corresponding to the 
source and target of the morphism. Composition of morphisms is obvious. The symmetric monoidal structure arises from the operation of disjoint union.

Let us now specialize to the case $D=1$. In this case the definition can be simplified, because all $1 \mathrm{~d}$ manifolds are orientable. Since we are given trivializations of $E$ at all base points, as well a trivialization of $o(M) \otimes \rho(E)$, we also have a trivialization of $o(M)$ at all base points. But since $M$ is orientable, this means that we are given a trivialization of $o(M)$ everywhere, i.e. an orientation. Then $\rho(E)$ is also trivialized everywhere, and the $G$-bundle reduces to a $G_{0}$-bundle. Thus the objects for $\mathcal{C}$ are exactly the same as in the oriented equivariant TQFT with symmetry group $G_{0}$. Morphisms are different however, for example because unorientable bordisms are now allowed. Moreover, even when bordisms are orientable, they are not given an orientation. More precisely, if the boundary of a bordism is connected, there is a base point with an orientation on it, and one can use this to extend orientation to the whole $N$. But if more than one base point is present, there is no guarantee that orientations so obtained agree between each other. This will be discussed in more detail below.

\subsection{Algebraic description for $D=1$}

From the above definition we extract the following algebraic data. First of all, let $M=S^{1}$. As remarked above, $S^{1}$ is actually oriented, and the structure group $G$ is reduced to $G_{0}$. Thus unoriented equivariant TQFT assigns a vector space $\mathcal{A}_{g}$ to every $g \in G_{0}$.

Now consider a cylinder regarded as a bordism from $S^{1}$ to $S^{1}$. It has two marked points on the boundaries which we call $p_{-}$and $p_{+}$(source and target). A $G$-bundle over a cylinder trivialized over $p_{-}$is determined by the holonomy around the source $S^{1}$ and thus is labeled by an element $g \in G$. We are also given a trivialization at $p_{+}$, and the holonomy along a path from $p_{-}$to $p_{+}$gives a well-defined element $h \in G$. We know that $g \in G_{0}$, but $h$ can be an arbitrary element of $G$. If $\rho(h)=1$, the two trivializations of $\rho(E)$ obtained from the trivializations of $E$ at $p_{-}$and $p_{+}$agree. Then, since $o(N) \otimes \rho(E)$ is trivialized everywhere, the orientations at $p_{-}$and $p_{+}$also agree, and the source and target circles have the same orientation. Thus the source is labeled by $g$, and the target by $h g h^{-1}$, and the cylinder is assigned a map $\alpha_{h}: \mathcal{A}_{g} \rightarrow \mathcal{A}_{h g h^{-1}}$. Similarly, if $\rho(h)=-1$, the two orientations disagree, and the target is labeled by $h g^{-1} h^{-1}$, while the source is still labeled by $g$. Such a cylinder is assigned a map $\alpha_{h}: \mathcal{A}_{g} \rightarrow \mathcal{A}_{h g^{-1} h^{-1}}$. We can summarize both cases by saying that $\alpha_{h}$ maps $\mathcal{A}_{g}$ to $\mathcal{A}_{h g^{\rho(g)} h^{-1}}$. Since gluing two cylinders labeled by $(g, h)$ and $\left(h g^{\rho(g)} h^{-1}, h^{\prime}\right)$ using the trivial identification of target and source circles gives a cylinder labeled by $\left(g, h^{\prime} h\right)$, we must have $\alpha_{h^{\prime}} \circ \alpha_{h}=\alpha_{h^{\prime} h}$. In particular, each $\alpha_{h}$ is invertible.

In general, we note that if $N$ is an orientable bordism, and the paths between base points on different boundary components all lie in $G_{0}$, the morphism becomes a morphism in the oriented equivariant theory with symmetry group $G_{0}$. Thus we get all the same algebraic data as in the oriented $G_{0}$-equivariant theory. That is, a $G_{0}$-crossed algebra

$$
\mathcal{A}=\oplus_{g \in G_{0}} \mathcal{A}_{g}, \quad \eta: \mathcal{A} \otimes \mathcal{A} \rightarrow \mathbb{C}, \quad \alpha: G_{0} \rightarrow \text { Aut } \mathcal{A},
$$


satisfying (2.3)-(2.8). In particular, for $h \in G_{0}$ the map $\alpha_{h}$ is an automorphism of $\mathcal{A}$. On the other hand, for $h \notin G_{0}$ the map $\alpha_{h}$ is an anti-automorphism:

$$
\alpha_{h}(a b)=\alpha_{h}(b) \alpha_{h}(a), \quad \forall h \notin G_{0}, \forall a, b \in \mathcal{A} .
$$

To see this, we compare the pants diagrams with cylinders attached either to the torso or to the pant legs and note that for $h \notin G_{0}$ they are related by a reflection rather than the identity homeomorphism.

Finally, in the unoriented case we have cross-cap states $\theta_{g} \in \mathcal{A}_{g^{2}}, g \notin G_{0}$. The state $\theta_{g}, g \notin G_{0}$, arises from a Möbius strip with an oriented boundary and a base point on the boundary. The fundamental group of the Möbius strip is isomorphic to $\mathbb{Z}$, where an orientation-reversing generator is fixed once the orientation of the boundary has been fixed. $\theta_{g}$ corresponds to a $G$-bundle whose holonomy along this generator is $g$.

The cross-cap states have the following properties:

$$
\begin{aligned}
& \alpha_{h \in G_{0}}\left(\theta_{g}\right)=\theta_{h g h^{-1}} \quad \text { and } \quad \alpha_{h \notin G_{0}}\left(\theta_{g}\right)=\theta_{h g^{-1} h^{-1}} \\
& \theta_{g} \cdot \psi_{k}=\alpha_{g}\left(\psi_{k}\right) \cdot \theta_{g k} \quad \text { for all } \quad \psi_{k} \in \mathcal{A}_{k} . \\
& \sum_{i} \alpha_{g}\left(\xi_{g h}^{i}\right) \xi_{i}^{g h}=\theta_{g} \cdot \theta_{h}
\end{aligned}
$$

The first of these properties is illustrated in figure 1. The vectors $\theta_{h g^{(-1)} h^{-1}}$ and $\alpha_{h}\left(\theta_{g}\right)$ are defined by the two pictures which happen to be related by an isotopy. The second property arises from an isotopy of the punctured Möbius strip shown in figure 2. The third property arises from the fact that a Klein bottle with two holes can be represented in two apparently different ways: as a cylinder with an orientation-reversing twist, or as a cylinder with an insertion of two cross-caps, see figure 3.

We will call the data $\left(\mathcal{A}, \eta, \alpha, \theta_{g}, g \notin G_{0}\right)$ a $\rho$-twisted $G$-crossed algebra.

Proposition 1. Unoriented equivariant $D=1$ TQFTs with symmetry $\left(G, \rho: G \rightarrow \mathbb{Z}_{2}\right)$ are in bijective correspondence with $\rho$-twisted $G$-crossed algebras $\left(\mathcal{A}, \eta, \alpha, \theta_{g}, g \notin G_{0}\right)$.

We have already explained how to assign this algebraic data to any unoriented equivariant $D=1$ TQFT. The converse procedure is described in appendix A.

\subsection{Invertible unoriented equivariant $D=1$ TQFT}

Let us now specialize to the invertible case. For an invertible unoriented equivariant $D=1$ TQFT, the vector spaces $\mathcal{A}_{g \in G_{0}}$ are one-dimensional. After fixing a basis $\left\{\ell_{g}\right\}_{g \in G_{0}}$ of $\mathcal{A}$ so that $\eta\left(\ell_{g}, \ell_{g^{-1}}\right)=1$, the $\rho$-twisted $G$-crossed algebra is determined by nonzero complex numbers $\theta(g), g \notin G_{0}, b(h, k), z(h, k), h, k \in G_{0}, w(h, k), h \notin G_{0}, k \in G_{0}$ defined as follows:

$$
\begin{aligned}
m_{k, l}\left(\ell_{k}, \ell_{l}\right) & =b(k, l) \ell_{k l}, & \theta_{g} & =\theta(g) \ell_{g^{2}}, \\
\alpha_{h \in G_{0}}\left(\ell_{k}\right) & =z(h, k) \ell_{h k h^{-1}}, & \alpha_{h \notin G_{0}}\left(\ell_{k}\right) & =w(h, k) \ell_{h k^{-1} h^{-1}} .
\end{aligned}
$$

These numbers satisfy a number of identities due to the properties of $\mathcal{A}$. 


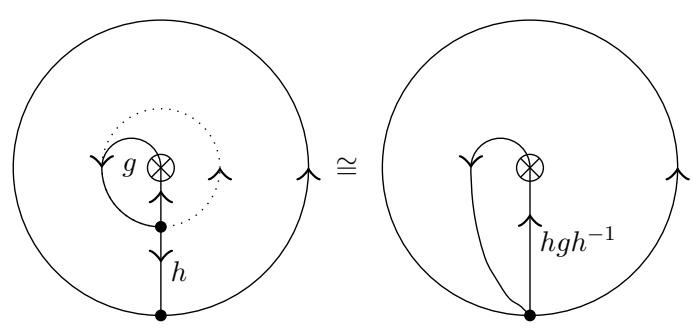

(a) $h \in G_{0}$.

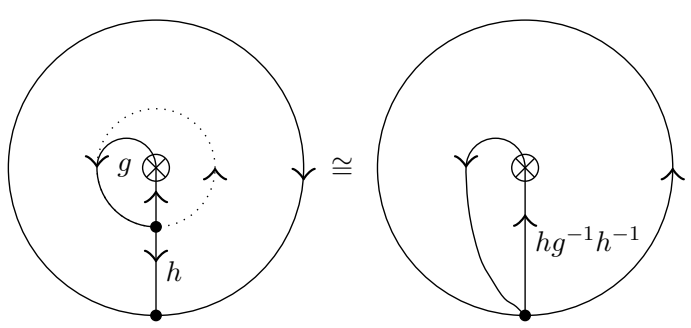

(b) $h \notin G_{0}$.

Figure 1. Axiom (4.3).

Proposition 2. Invertible unoriented equivariant $D=1$ TQFTs with symmetry $(G, \rho)$ are in bijective correspondence with elements of the $\rho$-twisted group cohomology $H^{2}\left(B G, \mathrm{U}(1)_{\rho}\right)$.

Twisted cohomology is the cohomology of the usual group cochain complex with respect to the $\rho$-twisted coboundary maps

$$
\delta_{\rho}^{n}: C^{n}(G, \mathrm{U}(1)) \rightarrow C^{n+1}(G, \mathrm{U}(1)) .
$$

In degree 2 , the $\rho$-twisted cocycle condition reads

$$
a(g, h) a(g h, k)=a(h, k)^{\rho(g)} a(g, h k) .
$$

A proof of proposition 2 is rather lengthy, see appendix B. But the map in one direction, from twisted group cohomology to the set of algebraic data (4.6), is easy to describe:

$$
\begin{aligned}
b(k, l) & =a(k, l) \\
\theta(g) & =a(g, g) \\
z(h, k) & =\frac{a(h, k) a\left(h k, h^{-1}\right)}{a\left(h, h^{-1}\right)} \\
w(h, k) & =\frac{a\left(h, k^{-1}\right) a\left(h k^{-1}, h^{-1}\right) a\left(k, k^{-1}\right)}{a\left(h, h^{-1}\right)} .
\end{aligned}
$$

To prove proposition 2, we must show that these numbers satisfy the TQFT axioms (4.2)(4.5) and that the map is injective and surjective.

\section{A Proof of proposition 1}

We have already shown that an unoriented equivariant $D=1$ TQFT with symmetry $(G, \rho)$ has an underlying $\rho$-twisted $G$-crossed algebra $\left(\mathcal{A}, \theta_{g}, \alpha_{h}\right)$. Oriented cobordisms and bundle isomorphisms constitute a $G_{0}$-crossed algebra $\mathcal{A}=\oplus_{g \in G_{0}} \mathcal{A}_{g}$, while crosscaps correspond to states $\theta_{g} \in \mathcal{A}_{g^{2}}$ and orientation-reversing homeomorphisms to algebra anti-automorphisms $\alpha_{h}: \mathcal{A}_{g} \rightarrow \mathcal{A}_{h g^{-1} h^{-1}}$. It remains to show the converse: that from each such algebra we can construct an unoriented equivariant TQFT with this underlying algebra. We generalize the approaches of [10] and [18] to unoriented equivariant theories. 


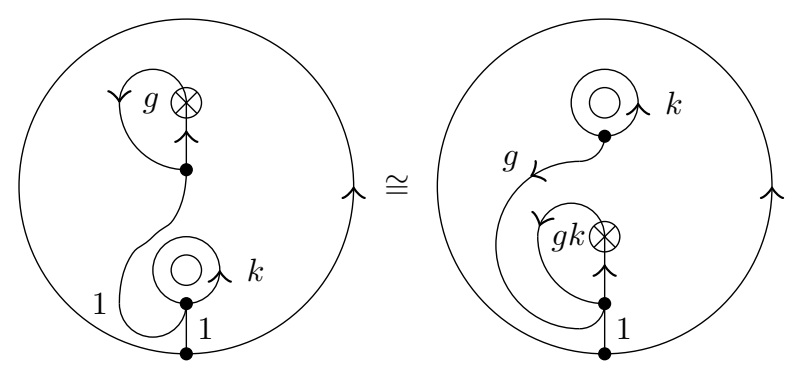

Figure 2. Axiom (4.4). To obtain the right figure from the left, the puncture with holonomy $k$ is pulled through the crosscap along the path with holonomy $g$.

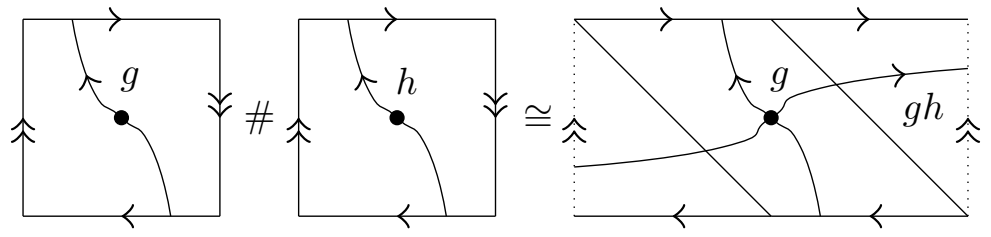

Figure 3. Axiom (4.5). Two projective planes are punctured and sewed along their boundaries the diagonal lines - to obtain their connected sum, the Klein bottle.

We begin by defining the vector spaces assigned to simple objects $P_{[g], x, t}$ of the source category $\mathcal{C}$. To each circle $S$ equipped with principal $G$-bundle $P_{[g]}$, basepoint $x$, local trivialization $t:\left.P_{[g]}\right|_{x} \rightarrow G$, and global trivialization of $o(S) \otimes \rho\left(P_{[g]}\right)$, assign the vector space $\mathcal{H}\left(P_{[g], x, t}\right) \cong \mathcal{A}_{g}$ where $g$ is the holonomy of $P_{[g]}$ around $S$ with respect to $x$ and $t$. Any object $E$ can be factored into simple objects $\sqcup_{i} P_{\left[g_{i}\right], x_{i}, t_{i}}$ and assigned a vector space $\mathcal{H}(E) \cong \otimes_{i} \mathcal{H}\left(P_{\left[g_{i}\right], x_{i}, t_{i}}\right)$. It is clear that $\mathcal{H}(E)$ does not depend on the factorization of $E$.

Next we consider the linear maps assigned to morphisms of simple objects. One type of morphism $\tilde{\alpha}_{k}: P_{[g], x, t} \rightarrow P_{[g], y, s}$ arises from an isomorphism $f$ of the bundle $P_{[g]}$ where $(y, s)=\left(f(x),\left(f^{-1}\right)^{*} t\right)$. Realized as its mapping cylinder, $f$ must have a global trivialization of $o(S \times I) \otimes \rho(f)$. Since $o(S \times I)$ is trivial, so must be $\rho(f)$, and so the holonomy of $P_{[g]}$ along a positive path from $(x, t)$ to $(y, s)$ is an element $k \in G_{0}$. We assign the linear map $\alpha_{k}: \mathcal{A}_{g} \rightarrow \mathcal{A}_{k g k^{-1}}$ to this morphism. The other type of morphism $\tilde{\alpha}_{h}: P_{[g], x, t} \rightarrow P_{\left[g^{-1}\right], y, s}$ arises from a bundle anti-isomorphism $P_{[g]} \rightarrow P_{\left[g^{-1}\right]}$ whose restriction to the base circle is not isotopic to the trivial homeomorphism. Since a bundle map of this type exchanges the sheets of $o(S)$, the holonomy of $P_{\left[g^{-1}\right]}$ from $(x, t)$ to $(y, s)$ is an element $h \notin G_{0}$. We assign the linear map $\alpha_{h}: \mathcal{A}_{g} \rightarrow \mathcal{A}_{h g^{-1} h^{-1}}$ to $\tilde{\alpha}_{h}$. This assignment is well defined for isomorphism classes of bundles, as the cylinder $\tilde{\alpha}_{k} \tilde{\alpha}_{g}$, related to $\tilde{\alpha}_{k}$ by a Dehn twist, is assigned the linear map $\alpha_{k} \alpha_{g}$, which equals $\alpha_{k}$ when restricted to $\mathcal{A}_{g}$ by (2.6).

Now we wish to define linear maps for cobordisms $\left(W, E_{0}, E_{1}\right)$. The strategy will be to decompose $W$ as a sequence of $n$ elementary cobordisms $\left(W^{i}, E_{0}^{i}, E_{1}^{i}\right)$, sewn along bundle (anti-)isomorphisms $s_{i}: E_{1}^{i} \rightarrow E_{0}^{i+1}$ with $E_{0}^{0}=E_{0}$ and $E_{1}^{n}=E_{1}$. After assigning a linear map to each $W^{i}$, we assign their composition $\tau(W)$ to $W$. We must then verify that $\tau(W)$ does not depend on the decomposition. Begin by considering the cobordism of base spaces $\left(N, M_{0}, M_{1}\right)$. By Sard's lemma, there exists a smooth function $f: N \rightarrow I$ such that $f^{-1}(0)=M_{0}, f^{-1}(1)=M_{1}$, and $f$ is Morse; that is, the gradient $d f$ vanishes at finitely many critical points $x_{i}$, the Hessian $d^{2} f$ is a non-degenerate quadratic form 
at all $x_{i}$, and the critical values $c_{i}=f\left(x_{i}\right)$ are distinct and not equal to 0 or 1 . The index $\operatorname{ind}\left(x_{i}\right)$ is the number of negative eigenvalues of $d^{2} f$ at $x_{i}$. Choose $t_{i} \in I$ such that $0=t_{0}<c_{1}<t_{1}<\cdots<c_{n}<t_{n}=1$. By the implicit function theorem, each $M_{t_{i}}=f^{-1}\left(t_{i}\right)$ is a disjoint union of $m_{i}$ circles, and $\Sigma_{i}=f^{-1}\left(\left[t_{i-1}, t_{i}\right]\right)$ is a cobordism from $M_{t_{i-1}}$ to $M_{t_{i}}$ with a single critical point. The classification of surfaces tells us that $\Sigma_{i}$ is homeomorphic to a disjoint union of cylinders and one of five possibilities: a cap, a pair-of-pants, their adjoints, and a twice-punctured real projective plane.

These spaces are base spaces for five classes of cobordisms $W$. Since any $G$-bundle over the disk is trivial, there is a unique cobordism over the cap, to which we assign the linear map $\eta: \mathcal{A}_{1} \rightarrow \mathbb{C}$. A $G$-bundle over the pair-of-pants, based and trivialized at the critical point, is almost determined by the holonomies $k$ and $l$ around the legs of the pants. We assign to it the linear map $m_{k, l}: \mathcal{A}_{k} \otimes \mathcal{A}_{l} \rightarrow \mathcal{A}_{k l}$. The orderings are related by conjugation $\alpha_{l}: \mathcal{A}_{k l} \rightarrow \mathcal{A}_{l k}$, and consistency requires that $m_{k, l}\left(\psi_{k} \otimes \psi_{l}\right)=\alpha_{k} m_{l, k}\left(\psi_{l} \otimes \psi_{k}\right)$, which is enforced by the axioms (2.6) and (2.7) of the $G_{0}$-crossed algebra $\mathcal{A}$. The holonomies determine the bundle up to cylinders $\tilde{\alpha}_{k}$ sewn to the boundary circles, which were assigned maps $\alpha_{k}$ above. The next two maps are fixed by adjunction. The adjoint of $\eta$ distinguishes a state $\psi_{\eta} \in \mathcal{A}_{1}$ with the property that $\eta\left(\psi_{\eta}\right)=1$. The adjoint pair-of-pants is assigned a map $\Delta_{k, l}\left(\psi_{k l}\right)=\sum_{i} \psi_{k l} \phi^{i} \otimes \phi_{i}$ where $\left\{\phi^{i}\right\}$ is a basis for $\mathcal{A}_{l}$ and $\left\{\phi_{i}\right\}$ is a dual basis for $\mathcal{A}_{l^{-1}}$. A $G$-bundle over the crosscap is specified (up to cylinders) by a holonomy $g \notin G_{0}$ around the orientation-reversing loop. We assign to it the linear map $\psi_{k} \mapsto m_{g^{2}, k}\left(\theta_{g} \otimes \psi_{k}\right)$, determined by the distinguished state $\theta_{g} \in \mathcal{A}_{g^{2}}$.

One may worry about a redundancy in the assignment of linear maps to composite cobordisms. Whenever an elementary cobordism $W^{i}$ and its sewing maps $s_{i-1}$ and $s_{i}$ can be modified in a way that preserves the composite cobordism $W$, consistency requires that $\tau(W)$ is also preserved. The map $s_{i}$ used to sew a cap or its adjoint into another cobordism does not affect the composite cobordism. The consistency of the algebraic description follows from the fact that $\alpha_{k}$ and $\alpha_{h}$ preserve $\eta$. Let $W^{i}$ be a pair-of-pants sewn along $s_{i-1}$ and $s_{i}$. Sewing instead along $\left(\tilde{\alpha}_{k} \otimes \tilde{\alpha}_{k}\right) \circ s_{i-1}$ and $s_{i} \circ \tilde{\alpha}_{k}^{-1}$ does not change $W$. Since $\alpha_{k}$ is an automorphism of $\mathcal{A}, \tau(W)$ is also preserved. Let $R$ be the bundle isomorphism that exchanges two circles. Then $\left(\tilde{\alpha}_{h} \otimes \tilde{\alpha}_{h}\right) \circ R \circ s_{i-1}$ and $s_{i} \circ \tilde{\alpha}_{h}^{-1}$ yield the same $W$. We require $\alpha_{h}^{-1} m\left(\alpha_{h}\left(\psi_{l}\right) \otimes \alpha_{h}\left(\psi_{k}\right)\right)=m_{k, l}\left(\psi_{k} \otimes \psi_{l}\right)$, which is enforced by axiom (4.2). Let $\left(W^{i}, E_{0}^{i}, E_{1}^{i}\right)$ be a twice-punctured real projective plane with holonomy $g$ realized as a cobordism from $s_{i-1}: P_{[k]} \rightarrow E_{0}^{i}$ to $s_{i}: E_{1}^{i} \rightarrow P_{\left[g^{2} k\right]}$. There is a bundle isomorphism, covering a Dehn twist of the base space, between this cobordism and a twice-punctured real projective plane with holonomy $g^{-1} k^{-1}$ with sewing maps $s_{i-1}$ and $s_{i} \circ \tilde{\alpha}_{g}$. By axioms (4.2) and (4.4), the consistency condition $\alpha_{g} m\left(\theta_{g^{-1} k^{-1}} \otimes \psi_{k}\right)=m_{g^{2}, k}\left(\theta_{g} \otimes \psi_{k}\right)$ is fulfilled. Now consider the Möbius strip with holonomy $g \notin G_{0}$ constructed by sewing a cap into the twice-punctured real projective plane with holonomy $g$. Sewing this cobordism into another along $s_{i}$ yields the same composite cobordism related to the Möbius strip with holonomy $h g^{-1} h^{-1}$ sewn along $\tilde{\alpha}_{h^{-1}} \circ s_{i}$ by a bundle isomorphism that covers a Y-homeomorphism of the base space. Axiom (4.3) encodes this relation in the algebraic data.

The linear map $\tau(W)$ assigned to an arbitrary cobordism $W$ is given by the composition of maps assigned to its factors under Morse decomposition. It remains to show that $\tau(W)$ 
does not depend on the choice of Morse function. Any two Morse functions $f_{0}$ and $f_{1}$ are related by a smooth family of functions $f_{s}$ that are Morse at all but finitely many values of $s$. One possibility is that two critical points merge and annihilate for some $s$. Then $f_{s}$ has a degenerate critical point. This situation only occurs when deforming a pair-of-pants and an adjoint cap into a cylinder. For $\tau(W)$ to be consistent over the deformation, we require $m_{k, 1}\left(\psi_{k} \otimes \psi_{\eta}\right)=\psi_{k}$. This condition is enforced by the axioms of $\mathcal{A}$. The remaining possibility is that two critical values coincide for some non-Morse value of $s$. We must check, for each composition $\mathrm{W}$ of two elementary cobordisms, that all factorizations give the same linear map. This situation occurs when both critical points have index 1 , in which case $W$ has Euler characteristic $\chi(W)=\sum_{i}(-1)^{\operatorname{ind}\left(x_{i}\right)}=-2$. Hence $W$ is one of seven cobordisms: a genus zero oriented cobordism from three circles to one, its adjoint, a genus zero oriented cobordism from two circles to two, a twice-punctured torus from one circle to one, a crosscap-pants cobordism from two circles to one, its adjoint, and a twice-punctured Klein bottle from one circle to one.

The consistency of the first two cobordisms follows immediately from associativity of multiplication. The remaining two oriented conditions have been proven in appendix A.3 of [10] and follow from the oriented axioms, notably (2.8). The next condition says that moving a crosscap from the "torso" to a leg of the pair-of-pants is a consistent deformation and also follows from associativity of multiplication. The Klein bottle has a decomposition as a pair-of-pants glued along its two legs to an adjoint pair-of-pants as well as a decomposition as a sphere with two crosscaps. The composite linear maps assigned to these realizations are equal to the others by axiom (4.5). We have assigned a linear map to each cobordism in terms of a Morse function $f$ and have seen that this map is independent of the choice of $f$. This completes the proof of proposition 1 .

\section{B Proof of proposition 2}

Consider the map from 2-cochains $a \in C^{2}(G, \mathrm{U}(1))$ to TQFT data defined in (4.9)-(4.12). If we restrict to the set $Z^{2}\left(G, \mathrm{U}(1)_{\rho}\right)$ of 2-cochains satisfying the $\rho$-twisted 2-cocycle condition (4.8), we obtain a map $f$ from twisted cocycles to TQFT data. We show that numbers in the image of $f$ satisfy axioms (4.2)-(4.5), and so give rise to a consistent invertible unoriented equivariant TQFT.

For an invertible theory, these axioms can be written as

$$
\begin{aligned}
w(h, k l) b(k, l) & =w(h, k) w(h, l) b\left(h l^{-1} h^{-1}, h k^{-1} h^{-1}\right) \\
w\left(h, g^{2}\right) \theta(g) & =\theta\left(h g^{-1} h^{-1}\right) \\
b\left(g^{2}, k\right) \theta(g) & =b\left(g k^{-1} g^{-1}, g k g k\right) w(g, k) \theta(g k) \\
b\left(g^{2} h g^{-1}, g h\right) w\left(g, h^{-1} g^{-1}\right) & =\theta(g) \theta(h) b\left(g^{2}, h^{2}\right) b\left(h^{-1} g^{-1}, g h\right) .
\end{aligned}
$$

It will be useful to impose a "cyclic-symmetric gauge" on the restriction of the cocycle $a$ to $G_{0}$ :

$$
a\left(k, k^{-1}\right)=1, \quad a(k, l)=a\left(l^{-1}, k^{-1}\right)^{-1}, \quad \forall k, l \in G_{0} .
$$

We also fix some $T \in G$ and impose the condition $a(k, T)=1, k \in G_{0}$. 
Axiom (4.2):

$$
\begin{aligned}
w(h, k l) b(k, l) & =\frac{a\left(h, l^{-1} k^{-1}\right) a\left(h l^{-1} k^{-1}, h^{-1}\right) a\left(k l, l^{-1} k^{-1}\right)}{a\left(h, h^{-1}\right)} a(k, l) \\
& =\frac{a\left(h, l^{-1} k^{-1}\right)}{a\left(h, h^{-1}\right)} \frac{a\left(h l^{-1}, k^{-1} h^{-1}\right)}{a\left(l^{-1}, k^{-1}\right) a\left(h l^{-1}, k^{-1}\right) a\left(k^{-1}, h^{-1}\right)} \\
& =\frac{a\left(h l^{-1}, k^{-1} h^{-1}\right)}{a\left(h, h^{-1}\right) a\left(k^{-1}, h^{-1}\right)} a\left(h, l^{-1}\right) \\
& =\frac{a\left(h, l^{-1}\right)}{a\left(h, h^{-1}\right) a\left(k^{-1}, h^{-1}\right)} \frac{a\left(h l^{-1} h^{-1}, h k^{-1} h^{-1}\right) a\left(h, k^{-1} h^{-1}\right)}{a\left(h l^{-1} h^{-1}, h\right)} \\
& =\frac{a\left(h, l^{-1}\right) a\left(h l^{-1} h^{-1}, h k^{-1} h^{-1}\right) a\left(h, k^{-1} h^{-1}\right)}{a\left(h, h^{-1}\right) a\left(k^{-1}, h^{-1}\right)} a\left(h^{-1}, h\right) a\left(h l^{-1}, h^{-1}\right) \\
& =\frac{a\left(h, l^{-1}\right) a\left(h l^{-1}, h^{-1}\right) a\left(h l^{-1} h^{-1}, h k^{-1} h^{-1}\right)}{a\left(h, h^{-1}\right) a\left(h, h^{-1}\right)} a\left(h, k^{-1}\right) a\left(h k^{-1}, h^{-1}\right) \\
& =w(h, k) w(h, l) b\left(h l^{-1} h^{-1}, h k^{-1} h^{-1}\right) .
\end{aligned}
$$

Axiom (4.3):

$$
\begin{aligned}
w\left(h, g^{2}\right) \theta(g) & =\frac{a\left(h, g^{-2}\right) a\left(h g^{-2}, h^{-1}\right) a\left(g^{2}, g^{-2}\right)}{a\left(h, h^{-1}\right)} a(g, g) \\
& =\frac{a\left(h g^{-2}, h^{-1}\right)}{a\left(h, h^{-1}\right)} a\left(h g^{-1}, g^{-1}\right) a\left(h, g^{-1}\right) a(g, g) a\left(g^{-1}, g^{-1}\right) \\
& =\frac{a\left(h, g^{-1}\right) a(g, g) a\left(g^{-1}, g^{-1}\right)}{a\left(h, h^{-1}\right)} a\left(g^{-1}, h^{-1}\right) a\left(h g^{-1}, g^{-1} h^{-1}\right) \\
& =\frac{a\left(h g^{-1} h^{-1}, h g^{-1} h^{-1}\right) a\left(h, g^{-1}\right) a(g, g) a\left(g^{-1}, g^{-1}\right)}{a\left(h, h^{-1}\right) a\left(h g h^{-1}, h\right) a\left(h, g^{-1} h^{-1}\right)} a\left(g^{-1}, h^{-1}\right) \\
& =\frac{a\left(h g^{-1} h^{-1}, h g^{-1} h^{-1}\right) a(g, g) a\left(g^{-1}, g^{-1}\right)}{a\left(h, h^{-1}\right) a\left(h g h^{-1}, h\right) a\left(h g^{-1}, h^{-1}\right)} \frac{a\left(g^{-1}, h^{-1}\right)}{a\left(h, g^{-1}\right) a\left(g^{-1}, h^{-1}\right)} \\
& =a\left(h g^{-1} h^{-1}, h g^{-1} h^{-1}\right) \\
& =\theta\left(h g^{-1} h^{-1}\right)
\end{aligned}
$$

Axiom (4.4):

$$
\begin{aligned}
\theta(g) a\left(g^{2}, k\right) & =\theta(g) a\left(g^{2}, k\right) \frac{a(g, k) a\left(g, k^{-1}\right) a\left(k, k^{-1}\right)}{a\left(g, g^{-1}\right)} \frac{a\left(g k^{-1}, k\right)}{a\left(g^{-1}, g k\right)} \\
& =\theta(g) a\left(g^{2}, k\right) a(g, k) \frac{a\left(g, k^{-1}\right) a\left(g k^{-1}, g^{-1}\right)}{a\left(g, g^{-1}\right)} a\left(g k^{-1} g^{-1}, g k\right) \\
& =\frac{a\left(g, k^{-1}\right) a\left(g k^{-1}, g^{-1}\right)}{a\left(g, g^{-1}\right)} a(g, g k) a\left(g k^{-1} g^{-1}, g k\right) \\
& =a\left(g k^{-1} g^{-1}, g k g k\right) \frac{a\left(g, k^{-1}\right) a\left(g k^{-1}, g^{-1}\right)}{a\left(g, g^{-1}\right)} a(g k, g k) \\
& =b\left(g k^{-1} g^{-1}, g k g k\right) w(g, k) \theta(g k)
\end{aligned}
$$


Axiom (4.5):

$$
\begin{aligned}
b\left(g^{2} h g^{-1}, g h\right) w\left(g, h^{-1} g^{-1}\right) & =a\left(g^{2} h g^{-1}, g h\right) \frac{a(g, g h) a\left(g^{2} h, g^{-1}\right) a\left(h^{-1} g^{-1}, g h\right)}{a\left(g, g^{-1}\right)} \\
& =\frac{a(g, g h)}{a\left(g, g^{-1}\right)} \frac{a\left(g^{2} h, h\right)}{a\left(g^{-1}, g h\right)} a\left(h^{-1} g^{-1}, g h\right) \\
& =\frac{a\left(g^{2}, h\right) a(g, g) a(g, h)}{a\left(g, g^{-1}\right) a\left(g^{-1}, g h\right)} a\left(g^{2} h, h\right) a\left(h^{-1} g^{-1}, g h\right) \\
& =\frac{a(g, g) a(g, h)}{a\left(g, g^{-1}\right) a\left(g^{-1}, g h\right)} a\left(g^{2}, h^{2}\right) a(h, h) a\left(h^{-1} g^{-1}, g h\right) \\
& =a(g, g) a(h, h) a\left(g^{2}, h^{2}\right) a\left(h^{-1} g^{-1}, g h\right) \\
& =\theta(g) \theta(h) b\left(g^{2}, h^{2}\right) b\left(h^{-1} g^{-1}, g h\right) .
\end{aligned}
$$

We have shown that data in the image of $f$ define consistent invertible unoriented equivariant TQFTs. Both $Z^{2}\left(G, \mathrm{U}(1)_{\rho}\right)$ and the set of invertible unoriented equivairant TQFTs are groups, and it is easy to see that $f$ is a group homomorphism.

It remains to show that $f$ is injective and surjective. Let $(g, h, k)$ denote the twisted cocycle condition (4.8). We construct a cocycle that solves (4.9)-(4.12), an inverse to $f$.

Consider the twisted cocycle condition for $\left(k, T, T^{-1}\right)$ :

$$
a(k, T) a\left(k T, T^{-1}\right)=a\left(T, T^{-1}\right) .
$$

Taking into account $a(k, T)=1$, we get $a\left(k T, T^{-1}\right)=a\left(T, T^{-1}\right)$. This also implies $a\left(T k, T^{-1}\right)=a\left(T, T^{-1}\right)$. So in this gauge we get $w(T, k)=a\left(T, k^{-1}\right)$. Next consider the twisted cocycle condition for $(l, k, T)$ :

$$
a(l, k) a(l k, T)=a(l, k T) a(k, T) .
$$

Taking into account $a(k, T)=1$, we get $a(l, k T)=a(l, k)$. Since $T^{-2} \in G_{0}$, this implies $a\left(k, T^{-1}\right)=a\left(k, T^{-2}\right)$. Next consider the twisted cocycle condition for $\left(T, k, T^{-1}\right)$ :

$$
a(T, k) a\left(T k, T^{-1}\right) a\left(k, T^{-1}\right)=a\left(T, k T^{-1}\right) .
$$

Using previous results, this is equivalent to

$$
a\left(T, k T^{-1}\right)=a\left(k, T^{-2}\right) a\left(T, T^{-1}\right) a(T, k) .
$$

Next consider the twisted cocycle condition for $(T, l, k)$ :

$$
a(T l, k) a(T, l) a(l, k)=a(T, l k) .
$$

Recall also that in our gauge $a(T, l)=w\left(T, l^{-1}\right)$. Then

$$
a(T l, k) a(l, k) w\left(T, l^{-1}\right)=w\left(T, k^{-1} l^{-1}\right) .
$$

Since $\alpha_{g} \alpha_{h}=\alpha_{g h}$ and by axiom (4.2), we see

$$
a(T l, k)=w\left(T, k^{-1}\right) a\left(T l T^{-1}, T k T^{-1}\right) .
$$


We have determined the components of the twisted cocycle where one argument is in $G_{0}$ and the other is not. We have also determined $a\left(T k, T^{-1}\right)$ and $a\left(T, k T^{-1}\right)$ up to a single term $a\left(T, T^{-1}\right)$. We can determine $a\left(T l, k T^{-1}\right)$ by requiring that $a$ satisfies the twisted cocycle condition $\left(T, l, k T^{-1}\right)$ :

$$
a\left(T l, k T^{-1}\right) a(T, l) a\left(l, k T^{-1}\right)=a\left(T, l k T^{-1}\right) .
$$

By construction, $a$ is a 2-cochain that satisfies (4.9)-(4.12) as well as the $\left(k, T, T^{-1}\right)$, $\left(l, k, T^{-1}\right),\left(T, k, T^{-1}\right),(T, l, k),(l, k, m)$, and $\left(T, l, k T^{-1}\right)$ cocycle conditions. The component $a\left(T l, m T^{-1}\right)$ is also determined by $\left(T l, k, T^{-1}\right)$, and equality of the two expressions must hold if $a$ is a cocycle:

$$
a\left(T l, k T^{-1}\right)=a\left(T l k, T^{-1}\right) a(T l, k) a\left(k, T^{-1}\right) .
$$

In the above expression, apply the $\left(T, l k, T^{-1}\right)$ condition to the first term to obtain $\frac{a\left(T, l k T^{-1}\right)}{a(T, l k) a\left(l k, T^{-1}\right)}$. Hit the second term with $(T, l, k)$ to obtain $\frac{a(T, l k)}{a(l, k) a(T, l)}$. Hit $a\left(l k, T^{-1}\right)$ with $\left(l, k, T^{-1}\right)$ to get $\frac{a\left(l, k T^{-1}\right) a\left(k, T^{-1}\right)}{a(l, k)}$. After cancellation, we are left with the first expression for $a\left(T l, k T^{-1}\right)$.

To see injectivity of $f$, consider the trivial TQFT with $b, w, \theta$ trivial. The cocycle solution has $a(k, l)=1$ and $a(k, l T)=1$. We have $a(T l, k)=\frac{w\left(T, k^{-1} l^{-1}\right)}{a(l, k) w\left(T, l^{-1}\right)}=1$ as well as

$$
a\left(T l, k T^{-1}\right)=a\left(T l k, T^{-1}\right) a(T l, k) a\left(k, T^{-1}\right)=\theta\left(T^{-1}\right)=1
$$

so the only the trivial cocycle corresponds to the trivial theory.

It remains to show that $a$ satisfies the cocycle condition for all possible combinations of arguments; in particular, we must show the $(k, l, m T),(k T, l, m),(k, l T, m),(k, l T, m T)$, $(k T, l, m T),(k T, l T, m)$, and $(k T, l T, m T)$ conditions. Consider the first condition:

$$
a(k, l) a(k l, m T)=a(l, m T) a(l, k m T) .
$$

Since $a(k, l T)=a(k, l)$ for all $k, l \in G_{0}$ in our gauge, this follows from the $G_{0}$ cocycle condition. Now consider the third:

$$
a(k T, l) a(k T l, m) a(l, m)=a(l T, k m) .
$$

Apply the $(T, k, l)$ condition to the first term to get $\frac{a(T, k l)}{a(k, l) a(T, k)}$, the $(T, k l, m)$ condition to the second term to get $\frac{a(T, k l m)}{a(k l, m) a(T, k l)}$, and the $(T, k, l m)$ condition to the third term to get $\frac{a(T, l k m) a(T, m)}{a(k, l m)}$. The desired condition is reduced to a known condition.

Now consider $\left(T k, l, m T^{-1}\right)$ :

$$
a(T k, l) a\left(T k l, m T^{-1}\right) a\left(l, m T^{-1}\right)=a\left(T k, l m T^{-1}\right) .
$$

The first term becomes $\frac{a(T, k l)}{a(T, k) a(k, l)}$ after $(T, k, l)$, the second $\frac{a\left(T, k l m T^{-1}\right)}{a(T, k l) a\left(k l, m T^{-1}\right)}$ after $\left(T, k l, m T^{-1}\right)$, the third $\left(a(k, l) a\left(k l, m T^{-1}\right)\right)^{-1}$ after $\left(l, m, T^{-1}\right)$, and the fourth $\frac{a\left(T, k l m T^{-1}\right)}{a(T, k) a\left(k, l m T^{-1}\right)}$ after $\left(T, k, l m T^{-1}\right)$. Everything cancels. 
Since $a\left(k T, T^{-1}\right)=a\left(T, T^{-1}\right)$, we get the $\left(l, l T, T^{-1}\right)$ condition by applying $\left(k l, T, T^{-1}\right)$ to $a\left(k l T, T^{-1}\right)$. Then $\left(k, l T, m T^{-1}\right)$ reads

$$
a(k, l T) a\left(k l t, m T^{-1}\right)=a\left(l T, m T^{-1}\right) a\left(k, l T m T^{-1}\right) .
$$

The last term is just $a(k, l T m)$ in our gauge and becomes $\frac{a(k, l T) a(k l T, m)}{a(l T, m)}$ after $(k, l T, m)$. $a\left(k l T, m T^{-1}\right)$ becomes $\frac{a\left(k l T m, T^{-1}\right) a(k l T, m)}{a\left(m, T^{-1}\right)}$ after $\left(k l T, m, T^{-1}\right)$, and $a\left(l T, m T^{-1}\right)$ becomes $\frac{a\left(l T m, T^{-1}\right) a(l T, m)}{a\left(m, T^{-1}\right)}$ after $\left(l T, m, T^{-1}\right)$. We have seen that $a\left(k l T m, T^{-1}\right)=a\left(T, T^{-1}\right)=$ $a\left(l T m, T^{-1}\right)$ so we are done.

The condition $\left(k, l T, T^{-1}\right)$ is shown by noting that $a(k, l T)=a(k, l)$ and $a\left(k l T, T^{-1}\right)=$ $a\left(T, T^{-1}\right)=a\left(l T, T^{-1}\right)$. Consider the $\left(k, T l, m T^{-1}\right)$ condition:

$$
a(k, T l) a\left(k T l, m T^{-1}\right)=a\left(T l, m T^{-1}\right) a\left(k, T l m T^{-1}\right) .
$$

Hit the second term with $\left(k T l, m, T^{-1}\right)$ to get $a\left(k T l m, T^{-1}\right) a\left(m, T^{-1}\right) a(k T l, m)$ and the fourth term with $\left(k, T l m, T^{-1}\right)$ to get $\frac{a\left(k T l m, T^{-1}\right) a(k, T l m)}{a\left(T l m, T^{-1}\right)}$. Then $a(k T l, m)$ becomes $\frac{a(k, T l m) a(T l, m)}{a(k, T L)}$ by $(k, T l, m)$ and $a\left(T l m, T^{-1}\right)$ becomes $\frac{a(T l, m) a\left(m, T^{-1}\right)}{a\left(T l, m T^{-1}\right)}$ by $\left(T, l m, T^{-1}\right)$.

Consider $(T, T, T)$ :

$$
a\left(T^{2}, T\right) a(T, T) a(T, T)=a\left(T, T^{2}\right) .
$$

The first term vanishes, and we are left with $\theta(T)^{2}=w\left(T, T^{-2}\right)$, which is true by axiom (4.5) with $g=h=T$.

Consider $\left(l T^{-1}, T, T\right)$ :

$$
a\left(l T^{-1}, T\right) a(T, T)=a\left(l T^{-1}, T^{2}\right) .
$$

The first term is just $\frac{a\left(T^{-1}, T\right)}{a\left(l, T^{-1}\right)}$ by $\left(l, T^{-1}, T\right)$. The third is $\frac{a\left(T^{-1}, T^{2}\right)}{a\left(l, T^{-1}\right)}$. The condition then follows from $(T, T, T)$. Consider $\left(T, m T^{-1}, T\right)$ :

$$
a\left(T, m T^{-1}\right)=a(T, m) a\left(m T^{-1}, T\right) .
$$

The first term becomes $a\left(T m, T^{-1}\right) a(T, m) a\left(m, T^{-1}\right)$ by $\left(T, m, T^{-1}\right)$ and the second becomes $\frac{a\left(T^{-1}, T\right)}{a\left(m, T^{-1}\right)}$. We are left with $a\left(T m, T^{-1}\right) a\left(T^{-1}, T\right)=1$. This is $\theta\left(T^{-1}\right) \theta(T)$, which vanishes by axiom (4.5). Now consider $\left(k T, l T^{-1}, T\right)$ :

$$
a\left(k T, l T^{-1}\right) a\left(l T^{-1}\right)=a(k T, l) .
$$

The first term is $a\left(k T l, T^{-1}\right) a(k T, l) a\left(l, T^{-1}\right)$ by $\left(k T, l, T^{-1}\right)$ and the second is $\frac{a\left(T^{-1}, T\right)}{a\left(l, T^{-1}\right)}$ by $\left(l, T^{-1}, T\right)$. We are left with $a\left(k T l, T^{-1}\right) a\left(T^{-1}, T\right)=1$ which holds as before.

Start with the $\left(T^{-1}, T, m T^{-1}\right)$ cocycle condition:

$$
a\left(T^{-1}, T\right) a\left(T, m T^{-1}\right)=a\left(T^{-1}, T m T^{-1}\right) .
$$

Apply $\left(T, m, T^{-1}\right)$ to the third term. It becomes $a\left(T m, T^{-1}\right) a(T, m) a\left(m, T^{-1}\right)$. Note that $a(T, m)=\frac{w\left(T, m^{-1}\right) a\left(T, T^{-1}\right)}{a\left(T m, T^{-1}\right)}$ and that $a\left(T^{-1}, T m T^{-1}\right)=\frac{w\left(T^{-1}, T m^{-1} T^{-1}\right) a\left(T^{-1}, T\right)}{a\left(m T^{-1}, T\right)}$. By $\left(m, T, T^{-1}\right)$, we have $a\left(m T^{-1}, T\right)=\frac{a\left(T^{-1}, T\right)}{a\left(m, T^{-1}\right)}$. The first equation becomes

$$
w\left(T, m^{-1}\right)=w\left(T^{-1}, T m^{-1} T^{-1}\right) .
$$


Since $\alpha_{T} \alpha_{T^{-1}}=1$, this becomes $w\left(T, m^{-1}\right) w(T, m)=1$ which is true by axiom (4.2). This proves the $\left(T^{-1}, T, m T^{-1}\right)$ cocycle condition.

Now consider the $\left(l T^{-1}, T, m\right)$ condition:

$$
a\left(l T^{-1}, T\right) a(l, m) a(T, m)=a\left(l T^{-1}, T m\right) .
$$

Hit the first term with $\left(l, T^{-1}, T\right)$ to get $\frac{a\left(T^{-1}, T\right)}{a\left(l, T^{-1}\right)}$ and the fourth term with $\left(l, T^{-1}, T m\right)$ to get $\frac{a(l, m) a\left(T^{-1}, T m\right)}{a\left(l, T^{-1}\right)}$. Apply the new result $\left(T^{-1}, T, m\right)$ to $a\left(T^{-1}, T m\right)$ to get $a(T, m) a\left(T^{-1}, T\right)$. Everything cancels. This proves $\left(l T^{-1}, T, m\right)$.

Now consider the $(l T, k T, m)$ condition:

$$
a(l T, k T) a(l T k T, m) a(k T, m)=a(l T, k T m) .
$$

Hit the first term with $(l T, k, T)$, the second term with the new result $(l T k, T, m)$, the third term with $(k, T, m)$, and the fourth term with $(l T, k, T m)$. Everything cancels.

Finally, check $\left(k T, T^{-1} l, m T\right)$ :

$$
a\left(k T, T^{-1} l\right) a(k l, m) a\left(T^{-1} l, m T\right)=a\left(k T, T^{-1} l m T\right) .
$$

The last term becomes $a\left(k T, T^{-1} l m\right) a\left(T^{-1} l m, T\right)$ by $\left(k T, T^{-1} l m, T\right) . \quad a\left(k T, T^{-1} l m\right)$ becomes $a(k l, m) a\left(k T, T^{-1} l\right) a\left(T^{-1} l, m\right)$ by $\left(k T, T^{-1} l, m\right)$ and $a\left(T^{-1} l m, T\right)$ becomes $\frac{a\left(T^{-1} l, m T\right)}{a\left(T^{-1} l, m\right)}$ by $\left(T^{-1} l, m, T\right)$. Everything cancels, proving the last cocycle condition $(k T, l T, m T)$.

This proves that each invertible unoriented equivariant TQFT arises from a twisted 2 -cocycle. Since this twisted 2-cocycle gives an inverse to $f$, we have shown that $f$ is surjective. This completes the proof of proposition 2 .

Open Access. This article is distributed under the terms of the Creative Commons Attribution License (CC-BY 4.0), which permits any use, distribution and reproduction in any medium, provided the original author(s) and source are credited.

\section{References}

[1] A. Kitaev, Homotopy-theoretic approach to SPT phases in action: $\mathbb{Z}_{16}$ classification of three-dimensional superconductors, talk given at Symmetry and Topology in Quantum Matter, Institute for Pure and Applied Mathematics, Los Angeles, U.S.A., 26-30 January 2015 http://helper.ipam.ucla.edu/publications/stq2015/stq2015_12389.pdf.

[2] X. Chen, Z.-C. Gu and X.-G. Wen, Local unitary transformation, long-range quantum entanglement, wave function renormalization and topological order, Phys. Rev. B 82 (2010) 155138 [arXiv: 1004.3835 ] [INSPIRE].

[3] X. Chen, Z.-C. Gu, Z.-X. Liu and X.-G. Wen, Symmetry protected topological orders and the group cohomology of their symmetry group, Phys. Rev. B 87 (2013) 155114 [arXiv: 1106.4772] [INSPIRE].

[4] A. Vishwanath and T. Senthil, Physics of three dimensional bosonic topological insulators: Surface Deconfined Criticality and Quantized Magnetoelectric Effect, Phys. Rev. X 3 (2013) 011016 [arXiv: 1209. 3058] [INSPIRE]. 
[5] A. Kapustin, Symmetry Protected Topological Phases, Anomalies and Cobordisms: Beyond Group Cohomology, arXiv: 1403.1467 [INSPIRE].

[6] D.S. Freed, Short-range entanglement and invertible field theories, arXiv:1406.7278 [INSPIRE].

[7] X. Chen, Z.-C. Gu and X.-G. Wen, Classification of gapped symmetric phases in $1 D$ spin systems, Phys. Rev. B 83 (2011) 035107 [arXiv: 1008.3745].

[8] L. Fidkowski and A. Kitaev, Topological phases of fermions in one dimension, Phys. Rev. B 83 (2011) 075103 [arXiv: 1008.4138].

[9] M. Atiyah, Topological quantum field theories, Inst. Hautes Etudes Sci. Publ. Math. 68 (1989) 175 [INSPIRE].

[10] G.W. Moore and G. Segal, D-branes and k-theory in $2 D$ topological field theory, hep-th/0609042 [INSPIRE].

[11] V. Turaev, Homotopy field theory in dimension two and group algebras, math.QA/9910010 [INSPIRE].

[12] G.W. Moore and N. Seiberg, Taming the Conformal Zoo, Phys. Lett. B 220 (1989) 422 [INSPIRE].

[13] B. Bakalov and A. Kirillov Jr., Lectures on Tensor Categories and Modular Functors, American Mathematical Society (2000).

[14] V. Turaev, Homotopy quantum field theory, in EMS Tracts in Mathematics, volume 10, EMS Publishing House, Zürich Switzerland (2010).

[15] V. Turaev and A. Virelizier, On 3-dimensional homotopy quantum field theory II: the surgery approach, Int. J. Math. 25 (2014) 1450027 [INSPIRE].

[16] V. Drinfeld, S. Gelaki, D. Nikshych and V. Ostrik, On braided fusion categories I, Selecta Math. (N.S.) 16 (2010) 1 [arXiv:0906.0620].

[17] H. Weyl, Commutator algebra of a finite group of collineations, Duke Math. J. 3 (1937) 200.

[18] V. Turaev and P. Turner, Unoriented topological quantum field theory and link homology, Algebr. Geomet. Topology 6 (2006) 1069 [math.GT/0506229] [INSPIRE].

[19] D.S. Freed and M.J. Hopkins, Reflection positivity and invertible topological phases, arXiv: 1605.06527 [INSPIRE].

[20] L. Bhardwaj, Unoriented 3d TFTs, arXiv:1611.02728 [INSPIRE]. 